\title{
Desafios e perspectivas da aprendizagem remota no contexto da pandemia COVID-19
}

\section{Challenges and perspectives of remote learning in the context of the COVID-19 pandemic}

Retos y perspectivas del aprendizaje a distancia em el contexto de la pandemia del COVID-19

Alexsandra Batista Cândido Siqueira

ORCID: https://orcid.org/0000-0002-8030-6450

Universidade Federal Rural do Semi-Árido, Brasil

E-mail: alexsandracandido@gmail.com

Alexandre Henrique Cavalcante da Costa

ORCID: https://orcid.org/0000-0002-2315-1122

Universidade Federal Rural do Semi-Árido, Brasil

E-mail: alexandre_hcc@hotmail.com

Suzana Virgínia da Costa Regis

ORCID: https://orcid.org/0000-0002-0282-8296

Universidade Federal Rural do Semi-Árido, Brasil

E-mail: suzanavdcr@hotmail.com

Yáskara Pryscilla Dantas Costa

ORCID: https://orcid.org/0000-0002-8713-7784

Universidade Federal Rural do Semi-Árido, Brasil E-mail: pryscilladantas@ hotmail.com

Juliana Carvalho de Sousa

ORCID: https://orcid.org/0000-0002-0388-3959

Universidade Federal Rural do Semi-Árido, Brasil

E-mail: juli.cs1009@gmail.com

Anderson Lopes Nascimento

ORCID: https://orcid.org/0000-0003-2538-9000

Universidade Federal do Piauí, Brasil

E-mail: adm.anderson@hotmail.com

\section{Resumo}

Este estudo teve como objetivo analisar a adesão dos professores, alunos e demais profissionais da educação quanto à implantação e andamento do ensino remoto durante a pandemia da COVID-19 no contexto do ensino superior. Tratase de uma pesquisa exploratória de natureza quantitativa, realizada no município de Mossoró, Estado do Rio Grande do Norte. A população de estudo foi composta por docentes, discentes e profissionais da educação do nível de coordenação ou supervisão. Para tanto, aplicou-se um questionário por meio eletrônico através da ferramenta Google forms. Os dados obtidos foram tabulados com o auxílio de planilhas eletrônicas e dispostos para apresentação dos resultados através de gráficos estatísticos e porcentagens. Em virtude de um baixo percentual de faltas, boa conectividade e manuseio dos aparelhos necessários para a manutenção da estabilidade das atividades nas aulas remotas, pode-se concluir que os estudantes e professores, bem como demais profissionais da educação superior de Mossoró - RN apresentaram aderência ao método de ensino à distância. Entretanto, verificou-se que apesar de aceitarem esse método de ensino como solução temporária ao período pandêmico, $67 \%$ dos respondentes informaram 
que seu aprendizado ficou prejudicado, enquanto $19 \%$ afirmaram ter permanecido igual, e apenas $14 \%$ dos respondentes afirmaram que melhorou.

Palavras-chave: Educação à distância; Tecnologias; Ensino-aprendizagem.

\begin{abstract}
This study aimed to analyze the adherence of teachers, students and other education professionals regarding the implementation and progress of remote education during the COVID-19 pandemic in the context of higher education. This is an exploratory research of a quantitative nature, carried out in the municipality of Mossoró, State of Rio Grande do Norte. The study population consisted of teachers, students and education professionals at the level of coordination or supervision. For that, a questionnaire was applied electronically through the Google forms tool. The data obtained were tabulated with the aid of electronic spreadsheets and arranged for presentation of results through statistical graphics and percentages. Due to a low percentage of absences, good connectivity and handling of basic services to maintain the stability of activities in remote classes, it is possible to fulfill that students and teachers, as well as other higher education professionals from Mossoró - RN student adherence to the distance learning method. However, it was found that despite accepting this teaching method as a temporary solution to the pandemic period, $67 \%$ of respondents reported that their learning was impaired, while $19 \%$ said they remained the same, and only $14 \%$ of respondents said it improved.
\end{abstract}

Keywords: Distance education; Technologies; Teaching-learning.

\title{
Resumen
}

Este estudio tuvo como objetivo analizar la adherencia de docentes, estudiantes y otros profesionales de la educación con respecto a la implementación y avance de la educación a distancia durante la pandemia COVID-19 en el contexto de la educación superior. Se trata de una investigación exploratoria de carácter cuantitativo, realizada en el municipio de Mossoró, Estado de Rio Grande do Norte. La población de estudio estuvo conformada por docentes, estudiantes y profesionales de la educación a nivel de coordinación o supervisión. Para eso, se aplicó un cuestionario de forma electrónica a través de la herramienta de formularios de Google. Los datos obtenidos se tabularon con la ayuda de planillas electrónicas y se dispusieron para la presentación de los resultados mediante gráficos estadísticos y porcentajes. Debido al bajo porcentaje de ausencias, buena conectividad y manejo de los dispositivos necesarios para mantener la estabilidad de las actividades en las clases remotas, se puede concluir que estudiantes y docentes, así como otros profesionales de la educación superior en Mossoró - RN mostraron adherencia a la método de aprendizaje a distancia. Sin embargo, se encontró que a pesar de aceptar este método de enseñanza como una solución temporal al período pandémico, el $67 \%$ de los encuestados informó que su aprendizaje se vio afectado, mientras que el 19\% dijo que seguía igual y solo el $14 \%$ de los encuestados dijo que mejoró.

Palabras clave: Educación a distancia; Tecnologías; Enseñanza-aprendizaje.

\section{Introdução}

A COVID-19 é uma síndrome respiratória aguda grave provocada pelo novo Coronavírus (SARS-COv-2). Essa doença surgiu em Wuhan, na China, e em três meses expandiu-se para mais de 118.000 casos, acarretando 4.291 mortes em 114 países. Como efeito, a Covid-19 foi classificada em 11 de Março de 2020 como uma pandemia pela Organização Mundial da Saúde (OMS), e tornou-se um dos maiores desafios sanitários deste século (Bavel et al., 2020).

A rápida disseminação da COVID-19 obrigou governos de todos os países a seguirem medidas preventivas de contenção do vírus, como quarentena e isolamento social, o que impactou diversos setores da sociedade, causando também uma crise no setor educacional (Flores \& Gago, 2020; He \& Harris, 2020; Nicola, 2020). Como consequência, houve o fechamento de escolas, faculdades e universidades; as aulas foram suspensas e todas as atividades de ensino foram canceladas ou adiadas (Jena, 2020). De acordo com as Organizações das Nações Unidades para a Educação, a Ciência e Cultura (Unesco, 2020), essas medidas afetaram cerca de 120 milhões de estudantes no mundo. A comunidade científica na área das doenças infecciosas atentou que o surgimento de novas pandemias não é uma questão de "se", mas de "quando" elas ocorrerão.

Para evitar uma crise ainda maior na educação, foi adotada como solução temporária, o ensino à distância (EAD), com auxílio das tecnologias de informação e comunicação (TICs) (Torres et al., 2020), como Skype, Youtube, GoogleMeet (Jena, 2020) e demais dispositivos de uso gratuito (Sepulveda-Escobar \& Morrison, 2020). 
No Brasil, a portaria MEC n 544, de 16 de junho de 2020, dispõe sobre a substituição das aulas presenciais por meios digitais enquanto durar a pandemia Covid-19, vedando a execução de disciplinas práticas ou de campo (Torres, Costa, \& Alves, 2020).

Nesse cenário, um dos maiores desafios é a adaptação do conteúdo e de metodologias ativas, a fim de garantir o sucesso do processo de aprendizagem (Flores \& Gago, 2020). Pelo exposto, embora as TICs possam auxiliar às novas formas de conhecimento, diversas instituições de ensino continuam resistentes em aderir ao EAD, de maneira mais específica, devido às limitações de acesso dos alunos a internet, o que pode prejudicar a qualidade de ensino e tornar este processo ineficiente (Andrade et al., 2020; Christian et al., 2020).

Estudos dessa natureza justificam-se pelo fato de realizar uma abordagem nos sujeitos envolvidos no processo de construção do conhecimento. Esta pesquisa tem por intuito analisar as vivencias dos profissionais da educação e estudantes do ensino superior quanto a utilização de ferramentas tecnológicas, a saber, tablets, smartphones, a própria internet nos seus processos de ensino-aprendizagem. Também será possível investigar sobre a aderência deste público ao ensino à distância e se estes possuem os aparatos tecnológicos necessários para manter o seu desenvolvimento estudantil enquanto durar o isolamento social imposto pelo período pandêmico.

Uma busca na literatura nacional e internacional demonstrou que são incipientes os estudos teóricos e empíricos que abordam os impactos da pandemia no contexto do ensino, sobretudo por se tratar de um tema novo e atemporal. Diante deste gap de pesquisa, o objetivo deste artigo é analisar a adesão dos professores, alunos e demais profissionais da educação quanto à implantação e andamento do ensino remoto durante a pandemia da COVID-19 no contexto do ensino superior.

\section{Desenvolvimento}

\subsection{O contexto da educação frente aos avanços tecnológicos}

A educação é uma área que vem passando por modificações ao longo dos anos, no que diz respeito ao processo de ensino e aprendizagem, e que tem reflexo direto na sociedade; e isso tudo tem ligação com as tecnologias, sejam elas mais recentes ou não. Essas transformações são de bases da sociedade que também passam por modificações, que por si, desenvolve e transforma a vida das pessoas, conforme explica Bueno e Gomes (2011, p. 54):

A educação reflete as transformações da base material da sociedade e, por isso, não está acima da sociedade, mas consiste em uma dimensão concreta da vida material e que se modela em consonância com as condições de existência dessa mesma sociedade.

Outro fator de modificação e evolução da educação é a tecnologia, em especial o advento da informática. Essa que trouxe várias mudanças nas mais diversas áreas do conhecimento, e com a educação não foi diferente. A informática contribui para a formação de muitas pessoas. Moraes (2002) explica que a informática tem papel de destaque no desenvolvimento das pessoas e nos processos construtivos de aprendizagem, com a criação de novos espaços virtuais, e novas formas de representação da realidade.

O Ensino a distância $(\mathrm{EaD})$ é anterior ao desenvolvimento da informática, pois no Brasil os primeiros registros da $\mathrm{EaD}$ foram por meio de cursos através do rádio, e por meio de cartas pelos correios conforme Silva (2001). O ensino EaD surge por lacunas do ensino presencial e por meio da exigência da sociedade que necessitam de meios alternativos para estudar, em que muitas pessoas não têm acesso ao ensino presencial ou não tem tempo, já que muitas pessoas trabalham ou tem outras ocupações.

Os meios alternativos de educação, como no caso do $\mathrm{EaD}$ fortalecem os processos de aprendizagem, com a chegada da informática há um avanço no ensino EaD no Brasil, Silva (2001, p.76) aponta que: 
É preciso considerar que as tecnologias - sejam elas novas (como o computador e a Internet) ou velhas (como o giz e a lousa) condicionam os princípios, a organização e as práticas educativas e impõem profundas mudanças na maneira de organizar os conteúdos a serem ensinados, as formas como serão trabalhadas e acessadas as fontes de informação, e os modos, individuais e coletivos, como irão ocorrer as aprendizagens.

$\mathrm{O} \mathrm{EaD}$ no Brasil cresce ao longo dos anos, e há uma crescente oferta de cursos superiores e de pós-graduação, e que segundo Niskier (2000) tornou-se uma modalidade fundamental de aprendizagem, mas que não é uma modalidade nova, apenas com o avanço da tecnologia, e mais especificamente da informática e da internet. Essa modalidade é diferente do ensino presencial em alguns aspectos, mas que tem com figura central os alunos e professores, mesmo que em uma sala de aula diferente.

No Brasil o EaD é uma modalidade de ensino regulamentada por lei específica, no ano de 2005 com o Decreto ${ }^{\circ}$ 5.622, as Tecnologias da Informação e Comunicação ou as TICs são incorporadas à definição de EAD, que passa a ser entendida como:

Modalidade educacional na qual a mediação didático-pedagógica nos processos de ensino e aprendizagem ocorre com a utilização de meios e tecnologias de informação e comunicação, com estudantes e professores desenvolvendo atividades educativas em lugares ou tempos diversos.

$\mathrm{O}$ avanço do $\mathrm{EaD}$ no Brasil ocorreu com o desenvolvimento e propagação das TICs que trouxeram uma nova modelagem ao ensino a distância e a educação. Segundo Almeida (2003) as práticas do ensino EaD são mais aceitáveis devido a flexibilidade do tempo empregado, bem como a quebra de barreiras, o rápido envio e recebimento de materiais, onde os mecanismos de transmissão e recebimento de informações são mais rápidos do que outros modelos de ensino presencial, as barreiras geográficas são facilmente superadas.

\section{Metodologia}

\subsection{Caracterização da pesquisa}

Trata-se de uma pesquisa do tipo exploratória com abordagem quantitativa (Pereira et al., 2018). Esta pesquisa foi desenvolvida em Mossoró, Estado do Rio Grande do Norte (RN) durante os meses de junho, julho e agosto de 2020. A população de estudo foi composta por docentes, discentes e profissionais da educação do nível de coordenação ou supervisão. Utilizou-se um procedimento de amostragem estratificada na qual obteve-se 575 respostas, sendo estas subdividas em 322 alunos, 207 professores e 46 coordenadores e/ou supervisores, os participantes da pesquisa eram de nível superior de instituições de natureza privada ou pública da cidade.

$\mathrm{O}$ instrumento de pesquisa deste trabalho foi um questionário aplicado através da plataforma Google forms $^{\circledR}$. O instrumento foi enviado aos respondentes via aplicativo de mensagens instantâneas WhastApp, no qual era precedido de um texto explicativo dos objetivos da pesquisa, bem como de uma rápida explanação sobre a importância dos resultados obtidos e do cuidado ao responder cada questionamento.

\subsection{Tabulação dos dados}

As respostas dos itens coletados através dos questionários junto aos docentes, discentes e coordenadores ou supervisores por meio do formulário eletrônico foram tabuladas mediante a transformação dos dados em porcentagens, e representados com o auxílio de planilhas eletrônicas através da construção de gráficos para representação destes resultados. 


\section{Resultados e Discussão}

De acordo com a Figura 1, verificou-se que este estudo obteve uma proporção de respondentes subdivididos da seguinte forma: $56 \%$ estudantes, $36 \%$ de professores e $8 \%$ de coordenadores e/ou supervisores.

Por haver uma maioria subdivida entre docentes e discentes, entende-se que os resultados desta pesquisa refletirão sobre a prática da sala de aula em si, pois ambos os públicos são os que mais estão fazendo uso das ferramentas tecnológicas durante o período da pandemia para assistir ou ministrar aula.

Figura 1 - Distribuição das proporções de respondentes da pesquisa. Mossoró - RN, 2020.

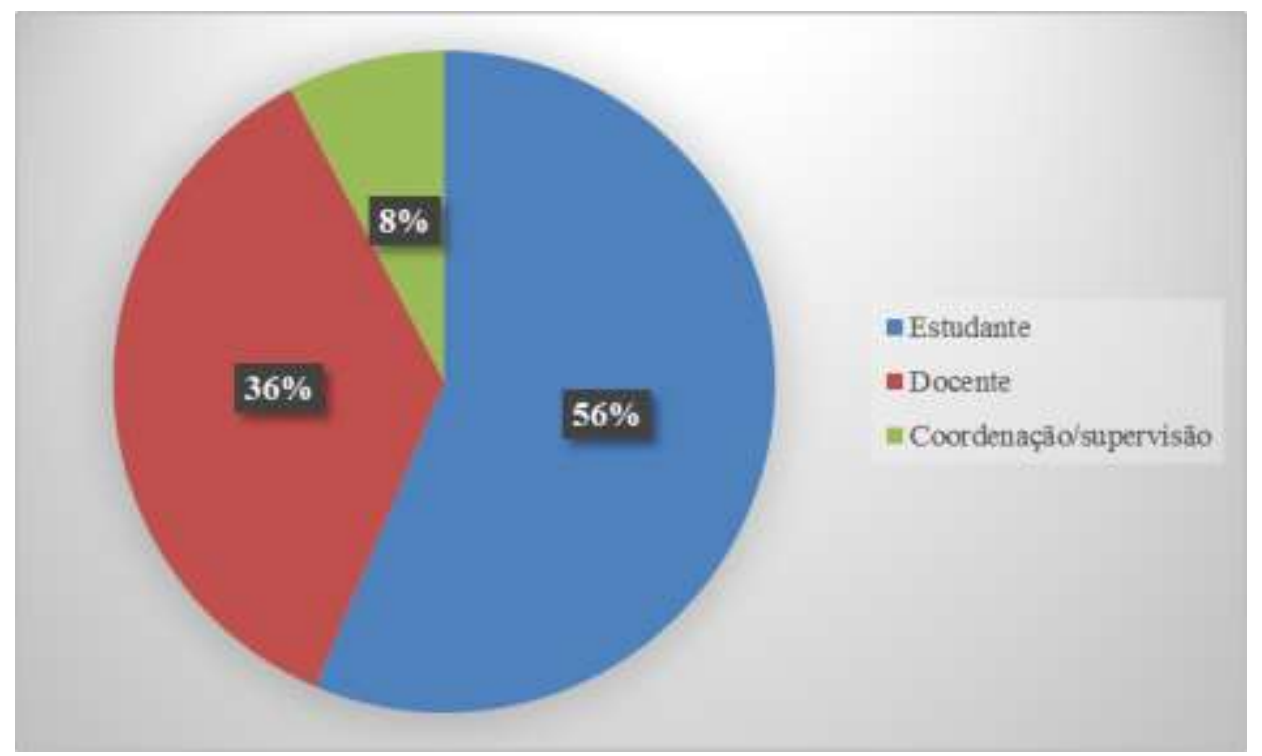

Fonte: Dados da pesquisa (2020).

Ao serem questionados sobre o grau de aprendizado durante o período da pandemia da COVID-19, 67\% dos respondentes informaram que ficou prejudicado, enquanto $19 \%$ afirmaram ter permanecido igual, e apenas $14 \%$ dos respondentes afirmaram que melhorou (Figura 2).

Esse resultado pode representar que os participantes desta pesquisa não sentem aderência pela modalidade de ensino à distância. Dentre os fatores que podem interferir nisso, pode-se mencionar, a dificuldade de adaptação às mudanças repentinas, sentimentos das pessoas envolvidas no processo, bem como dificuldades de manuseios dos aparelhos envolvidos nesse tipo de aula.

Com relação ao histórico de implantação do EaD no país, Silva et. al. (2004) apontaram que esta modalidade de ensino nasceu inicialmente como suporte ao ensino tradicional (presencial), e parecia ser uma excelente alternativa para as pessoas que viviam em regiões que não possuíam centros de estudos próximos de suas residências, especialmente os moradores de zonas rurais. Essas pessoas, na visão destes autores, acabavam por ficar excluídas do sistema educacional em virtude das limitações de distanciamento e econômicas.

Por outro lado, mesmo no ensino tradicional, há a percepção de que a tecnologia se encontra presente no processo de ensino dos dias atuais, mesmo na modalidade presencial. É cada vez mais comum a utilização de smartphones, tablets, aplicativos, softwares que modernizaram não só o processo do ensino como o método de o fazer. Entretanto, Fey (2012) entende que para esse tipo de ensino, principalmente no que diz respeito à utilização de novas tecnologias, tanto o professor quanto os alunos, precisam dominar a capacidade de utilização dos aparatos, e no caso dos professores, é necessário jogo de cintura para trazer concentração e motivação em suas aulas à distância de modo a incentivar os alunos a manter o foco. 
Figura 2 - Percepção sobre o grau de aprendizado durante o período de aulas remotas ao longo do isolamento social da pandemia COVID-19. Mossoró - RN, 2020.

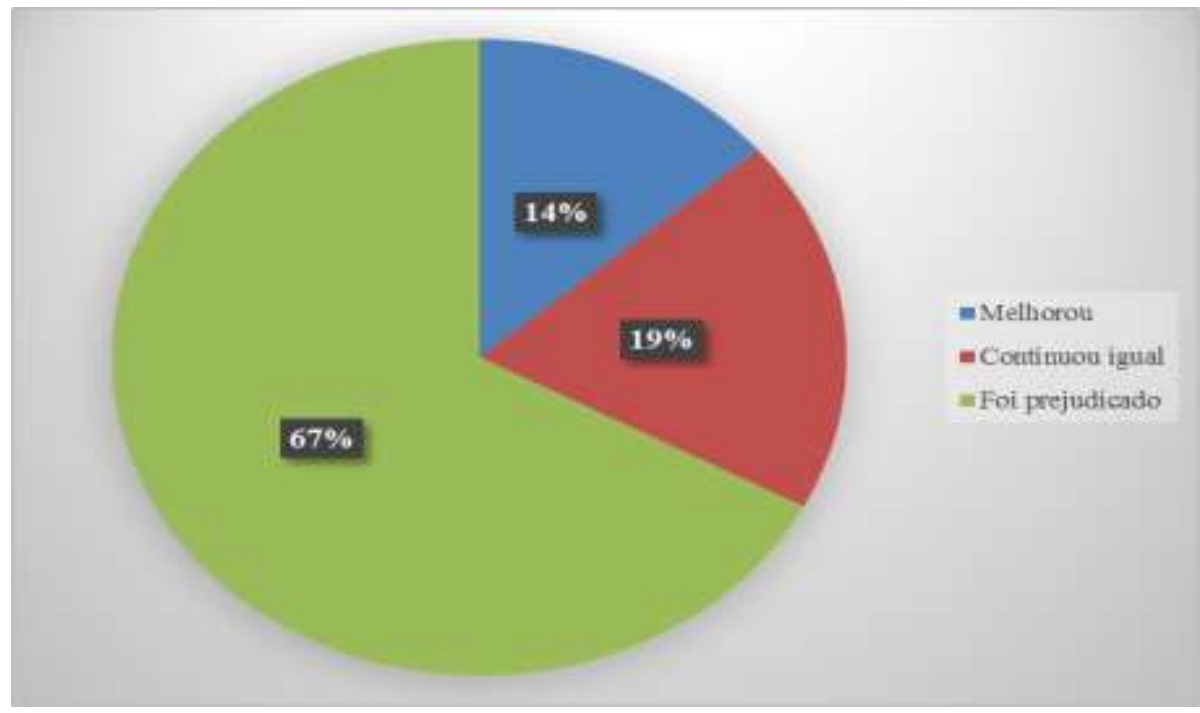

Fonte: Dados da pesquisa (2020).

Foi questionado se os professores apresentavam preparo para a realização das aulas remotas (Figura 3). Verificou-se que 59\% dos analisados neste estudo, concordam totalmente de que os professores estão aptos para atuar nessa modalidade, somente aproximadamente $10 \%$ dos respondentes ou discordam ou discordam totalmente dessa afirmação.

Este resultado dá a percepção de que os atores envolvidos no processo de ensino-aprendizagem na modalidade EaD reconhecem os esforços dos docentes para ajudar a manter a rotina de aulas e estudos, mesmo diante da pandemia e do isolamento.

De acordo com dados do Ministério da Educação (Brasil, 2006), a educação EaD tem sido uma estratégia para a formação de professores no Brasil; dessa forma, entende-se que se os docentes já tiveram contato com essa modalidade em suas formações continuadas, eles estarão mais aptos para aplicar ferramentas tecnológicas, bem como criar e implementar aulas mais atrativas para seus alunos.

Figura 3 - Percepção sobre a aptidão dos professores para as aulas à distância. Mossoró - RN, 2020.

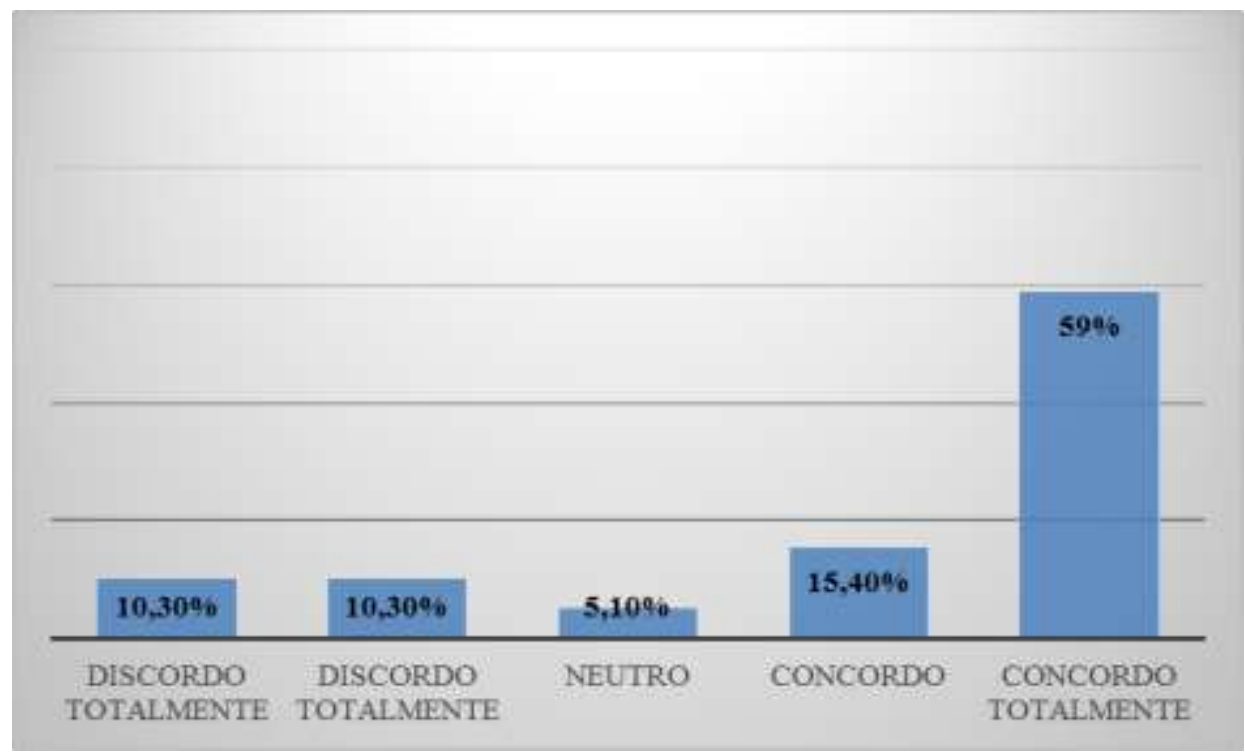

Fonte: Dados da pesquisa (2020). 
Foi questionado aos respondentes desta pesquisa se durante o isolamento social, eles haviam faltado às aulas à distância pelo fato de não estarem satisfeitos ou motivados para assisti-las. De acordo com a figura 4, verificou-se que 87,3\% afirmaram que não, enquanto $12,7 \%$ disseram sim. Mais uma vez, cabe ressaltar que o fato de a pesquisa ter obtido maior percentual de respostas entre alunos e professores (Figura 1), isso demonstra que esses indivíduos permaneceram comprometidos com o compromisso das aulas, mesmo que essa atividade tenha sofrido uma modificação do seu formato em função do período pandêmico.

Figura 4 - Porcentagem de falta às aulas em virtude de desmotivação ou insatisfação com a modalidade EaD. Mossoró - RN, 2020

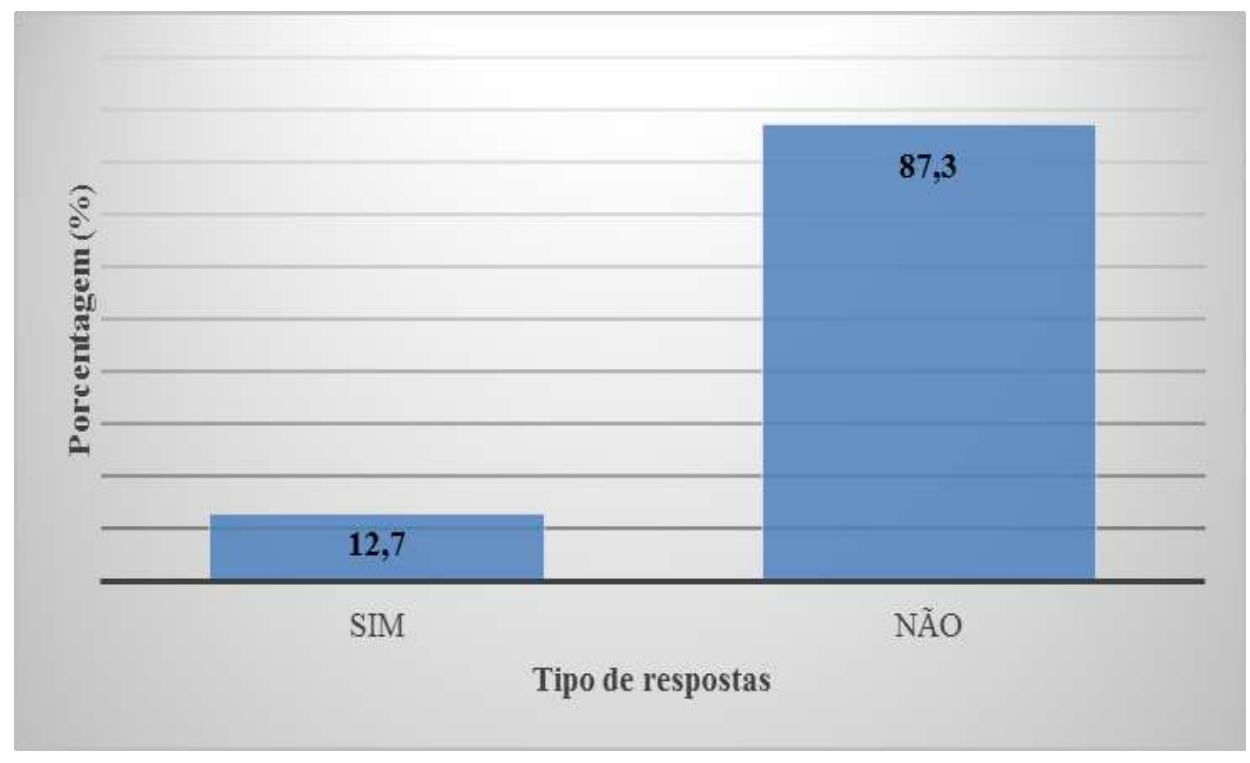

Fonte: Dados da pesquisa (2020).

A Figura 5 mostra a percepção dos indivíduos avaliados neste estudo quanto a visualização de melhorias nas estruturas das aulas. Neste caso, 95,5\% afirmaram não ter percebido melhoria nas estruturas das aulas, o que é um dado preocupante, em virtude de, neste tipo de modalidade de ensino, haver a necessidade de implementação do formato das aulas para que estas se tornem mais atrativas para os alunos e não ocorra dispersão destes.

Estudos de análise juntamente aos docentes e discentes, quanto ao detalhamento do formato da aula, o uso de metodologias ativas, as dificuldades técnicas e operacionais, permitirá a obtenção de elementos palpáveis para os formadores de professores. 
Figura 5 - Percepção de melhoria na estrutura das aulas. Mossoró - RN, 2020.

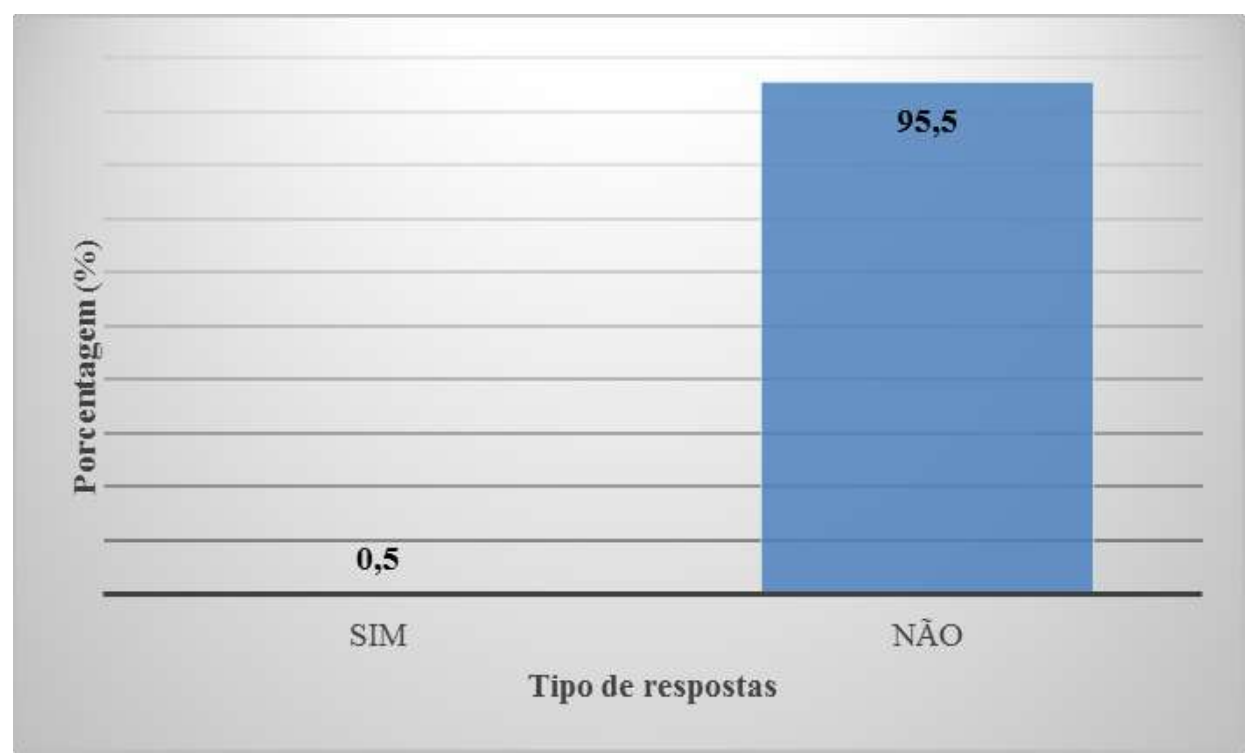

Fonte: Dados da pesquisa (2020).

Na Figura 6, foi questionado se os participantes dessa pesquisa tiveram problemas com atividades ou a própria aula em si, em virtude da funcionalidade dos seus aparelhos ou de sua conexão com a internet. Verificou-se que 18,2\% concordaram totalmente com essa afirmação, enquanto 35,5\% discordaram totalmente, juntamente com 25,5\% discordaram dessa afirmação. Esse dado demonstra que os analisados deste estudo não tiveram problemas em virtude dos seus aparelhos ou da conectividade, o que representa um dado positivo, tendo em vista a estrita dependência das aulas remotas do perfeito funcionamento desses mecanismos.

Este resultado discorda do encontrado por Cetric (2018), com relação à dimensão infraestrutura, um estudo realizado em 2018, pelo Centro Regional de Estudos para o Desenvolvimento da Sociedade da Informação do Brasil demonstrou que $30 \%$ dos domicílios brasileiros não possuem nem computador nem acesso à internet. Quando consideramos a renda familiar de até um salário-mínimo, esse percentual salta para 50\%, segundo a mesma pesquisa. Nesse cenário, necessitamos levar em consideração as desigualdades sociais no cenário brasileiro, visto que muitos discentes e docentes não dispõe da estrutura necessária para participarem da $\mathrm{EaD}$ em seus domicílios. Diante desse contexto as instituições privadas foram as pioneiras de ensino a distância antes das instituições públicas. Ou seja, o resultado deste estudo mostrou que a realidade dos atores relacionados ao processo educacional de ensino superior da cidade de Mossoró está melhor do que o parâmetro nacional, no que diz respeito ao acesso à internet e ao uso de TIC's. 
Figura 6 - Problemas com a funcionalidade de aparelhos e conexão de internet para o aproveitamento das aulas á distância. Mossoró - RN, 2020.

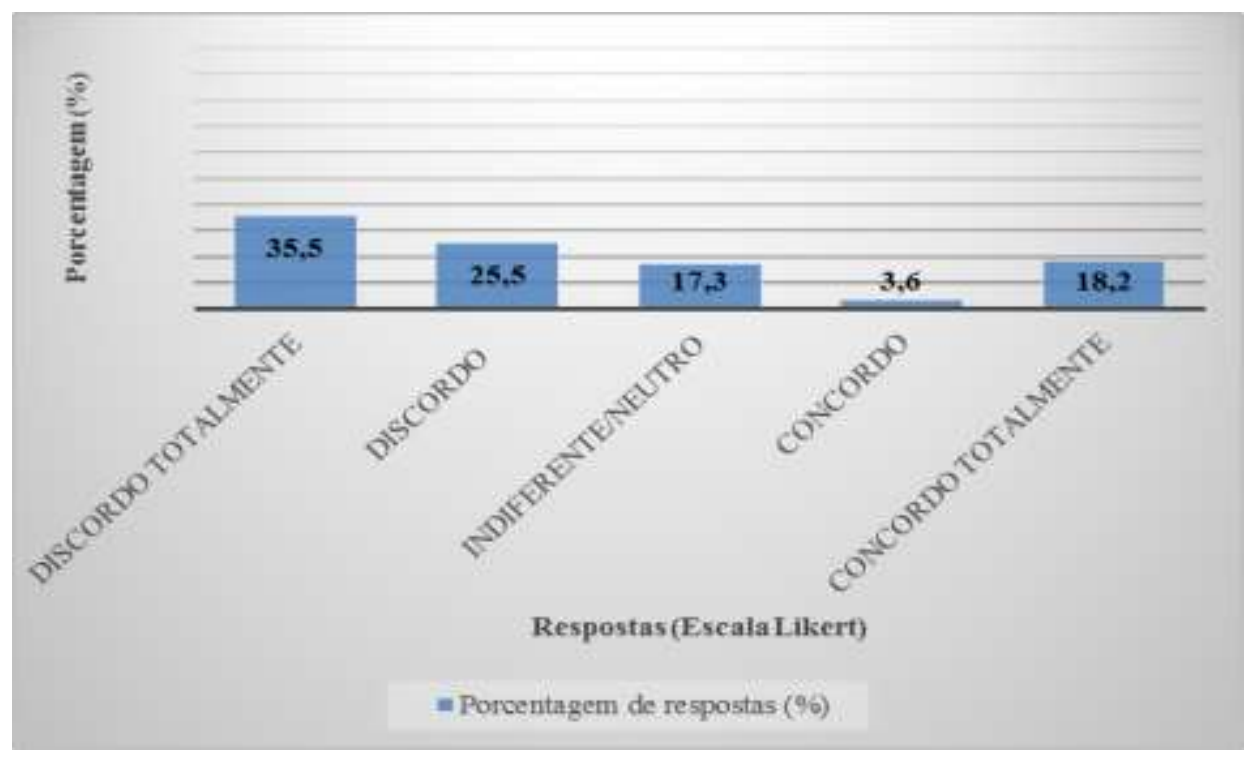

Fonte: Dados da pesquisa (2020).

\section{Conclusão}

Através deste estudo, verificou-se que os estudantes e profissionais da educação analisados nesta pesquisa reconhecem os esforços dos professores para manter o funcionamento e o fluxo de atividades nas aulas remotas durante o período da pandemia da COVID-19.

Apesar disso, verificou-se que a estrutura das aulas não sofreu alterações, o que demonstra que há a necessidade de implementação de técnicas pedagógicas para melhorar a aderência e o foco dos estudantes nesse formato de aulas.

Em virtude de um baixo percentual de faltas, boa conectividade e manuseio dos aparelhos necessários para a manutenção da estabilidade das atividades nas aulas remotas, pode-se concluir que os estudantes e professores, bem como demais profissionais da educação superior de Mossoró - RN apresentaram aderência ao método de ensino à distância.

Por último, apesar de aceitarem esse método de ensino como solução temporária ao período pandêmico, $67 \%$ dos respondentes informaram que seu aprendizado ficou prejudicado, enquanto $19 \%$ afirmaram ter permanecido igual, e apenas $14 \%$ dos respondentes afirmaram que melhorou.

Sugere-se para estudos futuros que seja realizada uma análise das principais fragilidades dos professores e alunos quanto ao manuseio dos aparatos tecnológicos como uma forma de melhorar o formato, a aderência e a aceitação da educação à distância, não mais como um complemento ao ensino tradicional, mas sim como uma modalidade que supra as necessidades dos estudantes que buscam qualificação.

\section{Referências}

Almeida, M. E. B. D. (2003). Educação à distância na internet: abordagens e contribuições dos ambientes digitais de aprendizagem. Educação $e$ pesquisa, 29(2), 327-340.

Andrade C. L., Rodrigues, W., França, G., \& Prata, D. N. (2020). Uso de tecnologias no ensino superior público brasileiro em tempos de pandemia COVID19. Research, Society and Development, 9(8), e267985485-e267985485. https://doi.org/10.33448/rsd-v9i8.5485

Brasil. Decreto ${ }^{\circ}$ 5.622, de 19 de dezembro de 2005. Regulamenta o art. 80 da Lei no 9.394, de 20 de dezembro de 1996 , que estabelece as diretrizes e bases da educação nacional. Diário Oficial da União de 20/12/2005. http://www.planalto.gov.br/ccivil_03/_ato2004-2006/2005/decreto/d5622.htm

Brasil. Decreto n. 5.800, de 8 de junho de 2006. Dispõe sobre o Sistema Universidade Aberta do Brasil - UAB. Brasília, 2006. http://www.planalto.gov.br/ccivil_03/_ato2004-2006/2006/decreto/d5800.htm\#: :text=DECRETA\%3A,de\%20educa\%C3\%A7\%C3\%A3o\%20superior\%20no $\% 20 \mathrm{~Pa} \% \mathrm{C} 3 \%$ ADs. 
Bueno, J. L. P., \& de Oliveira Gomes, M. A. (2011). Uma análise histórico-crítica da formação de professores com tecnologias de informação e comunicação. Revista Cocar, 5(10), 53-64.

Christian, D. D., McCarty, D. L., \& Brown, C. L. (2021). Experiential education during the COVID-19 pandemic: A reflective process. Journal of Constructivist Psychology, 34(3), 264-277. https://doi.org/10.1080/10720537.2020.1813666

Centro Regional de Estudos para o Desenvolvimento da Sociedade da Informação do Brasil. (2018). TIC em Domicílios, por presença de computador e internet. https://cetic.br/pt/tics/domicilios/2019/domicilios/A4B/

Diário Oficial da União. (2020). Dispõe sobre a substituição das aulas presenciais por aulas em meios digitais, enquanto durar a situação de pandemia do novo coronavírus - Covid-19, e revoga as Portarias MEC n 343, de 17 de março de 2020, nº 345, de 19 de março de 2020 , e nº 473 , de 12 de maio de 2020. https://www.ufrb.edu.br/ccaab/images/dire\%c3\%83\%e2\%80\%a1\%c3\%83\%c6\%92o/portaria_n\%c2\%ba_544_de_16_de_junho_de_2020_-

_portaria_n\%c2\%ba_544_de_16_de_junho_de_2020_-_dou_-_imprensa_nacional.pdf.

Fey, A. F. (2012). Dificuldades na transposição do ensino presencial para o ensino on-line. Anais do Seminário de pesquisa em educação da Região Sul, Caxias do Sul, RS, 9 .

Flores, M. A., \& Gago, M. (2020). Teacher education in times of COVID-19 pandemic in Portugal: national, institutional and pedagogical responses. Journal of Education for Teaching, 46(4), 507-516. https://doi.org/10.1080/02607476.2020.1799709

He, H., \& Harris, L. (2020). The impact of Covid-19 pandemic on corporate social responsibility and marketing philosophy. Journal of business research, 116, 176-182. https://doi.org/10.1016/j.jbusres.2020.05.030

Jena, P. K. (2020). Impact of pandemic COVID-19 on education in India. International journal of current research (IJCR), 12(7), 12582-12586. https://doi.org/10.24941/ijcr.39209.07.2020

Moraes, M. C. (2002). Tecendo a rede, mas com que paradigma. Educação à distância: fundamentos e práticas. Campinas, SP: UNICAMP/NIED, 1-12.

Nicola, M., Alsafi, Z., Sohrabi, C., Kerwan, A., Al-Jabir, A., Iosifidis, C., \& Agha, R. (2020). The socio-economic implications of the coronavirus pandemic (COVID-19): A review. International journal of surgery, 78, 185-193. https://doi.org/10.1016/j.ijsu.2020.04.018

Niskier, A. (2000). Educação à distância: a tecnologia da esperança. Loyola.

Pereira A. S. et al. (2018). Metodologia da pesquisa científica. UFSM.

Silva, M. (2001). Sala de aula interativa: a educação presencial e a distância em sintonia com a era digital e com a cidadania. Anais do Congresso Brasileiro de Comunicação, Campo Grande, 24.

Silva, T. A., Oliveira, F. A., Santos, C. M., Fonseca, C. D. G., Maia, F. A., \& Almeida, F. I. (2004). Uma Avaliação dos Desafios e Oportunidades da Educação à Distância. Qualitas - Revista eletrônica. 3(2).

Sepulveda-Escobar, P., \& Morrison, A. (2020). Online teaching placement during the COVID-19 pandemic in Chile: challenges and opportunities. European Journal of Teacher Education, 43(4), 587-607. https://doi.org/10.1080/02619768.2020.1820981

Torres, A. C. M., Alves, L. R. G., \& Costa, A. C. N. (2020). Educação e Saúde: reflexões sobre o contexto universitário em tempos de COVID-19. https://doi.org/10.1590/SciELOPreprints.640

United Nations Educational, Scientific and Cultural Organization - Unesco. (2020). Education: From disruption to recovery. https://en.unesco.org/covid19/educationresponse.

Van Bavel, J. J., Baicker, K., Boggio, P. S., Capraro, V., Cichocka, A., Cikara, M., \& Willer, R. (2020). Using social and behavioural science to support COVID-19 pandemic response. Nature human behaviour, 4(5), 460-471. https://doi.org/10.1038/s41562-020-0884-Z 